\title{
Semi-empirical extrapolation of JET baseline and hybrid scenario fusion performance to $\mathrm{D}-\mathrm{T}$ operation
}

\author{
H. WEISEN ${ }^{1}$, P. SIRÉN ${ }^{2,3}$, J. VARJE ${ }^{4,5}$, J. KILPELÄINEN ${ }^{4}$ and JET CONTRIBUTORS ${ }^{*}$ \\ ${ }^{1}$ Ecole Polytechnique Fédérale de Lausanne (EPFL), Swiss Plasma Center (SPC), Lausanne, Switzerland \\ ${ }^{2}$ Department of Physics, University of Helsinki, Helsinki, Finland \\ ${ }^{3}$ VTT Technical Research Centre of Finland, Espoo, Finland \\ ${ }^{4}$ Aalto University, Department of Applied Physics, Espoo, Finland \\ ${ }^{5}$ Tokamak Energy Ltd, Milton Park, OX14 4SD, United Kingdom
}

\begin{abstract}
* See the author list of "Overview of JET results for optimising ITER operation" by J. Mailloux et al. to be published in Nuclear Fusion Special issue: Overview and Summary Papers from the 28th Fusion Energy Conference (Nice, France, 10-15 May 2021)
\end{abstract}

\begin{abstract}
We present DT neutron rate extrapolations for the baseline and hybrid scenarios of the imminent JET DTE2 experimental campaign using the Monte Carlo heating code ASCOT, using empirically scaled plasmas parameters as inputs. The scalings used are based on the datasets that were produced during scenario development from 2016 to 2020. We find that the target fusion power of $15 \mathrm{MW}$ is likely to be achieved and even surpassed in hybrid scenarios if the same heating power is available as for the best discharges during scenario development. The extrapolated best baseline plasmas are also likely to achieve a fusion power close to that target.
\end{abstract}

\section{INTRODUCTION}

One of the main objectives of the second JET Deuterium (D)-Tritium (T) campaign (DTE2) is to establish pulses featuring a fusion power of $15 \mathrm{MW}$ for up to 5 seconds. To this effect the experimental teams for these scenarios have, from 2016 to 2020, been developing pulses in deuterium plasmas [1-4], the most promising of which are to be repeated in DTE2 in plasmas consisting of 50/50 D/T mixtures and heated mainly with Neutral Beam Heating (NBH), as well as with Ion Cyclotron Heating (ICRH). The total installed auxiliary power on JET for DT is about 40MW, of which $33.5 \mathrm{MW}$ from NBH to nearly equal parts D and $\mathrm{T}$ and the remainder by ICRH. In addition to plasma heating, ICRH serves to mitigate core impurity accumulation. The fusion power predicted for DTE2 in these scenarios by a variety physics based modelling is in the range 10-17 MW for a total input power of 40MW [2-4]. While the state-of-the-art modelling as in [2-4] includes all important known physics effects, the calculations are relatively time consuming and are limited to a narrow selection of discharges.

Empirical scaling of experimental data is a well known method for extrapolating plasma energy, momentum and particle confinement, es exemplified by the well known ITER scaling laws [5,6]. Direct empirical scaling of fusion neutron rates has been shown for DD neutrons in D plasmas [7]. However, directly extrapolating from DD neutron rates to DT neutron rates based purely on ratios of nuclear cross sections is likely to be unsatisfactory, as a realistic extrapolation needs at least to take into account the material changes to the heatings systems and fast ion species used to heat the thermal plasma. For NBH in JET this means converting half of the power to $\mathrm{T}$ injection, with a slightly lower injection energy (max $118 \mathrm{keV}$ for Tritium instead of max $125 \mathrm{keV}$ for D), which leads to lower neutral particle velocity and hence beam penetration (broader deposition profile) for Tritium. Moreover, the plasma parameters themselves are expected to change, with increased energy and likely particle confinement in DT $[6,8,9]$. Both scenarios have been developed towards a total input power near 40MW. The baseline scenario has additionally been developed towards a target plasma current of 4MA. This plasma current was achieved in a baseline in a single pulse, however without time for scenario optimisation at 4MA. The hybrid scenario aims at repeating the most promising D discharges at the same $\mathrm{I}_{\mathrm{p}}$ in DTE2, typically 2.3-2.5MA. The changed target conditions affect the beam deposition, the DT thermal and the beam-plasma neutron rates in different ways. In this paper we extrapolate from D reference plasmas to DT target plasmas by first empirically extrapolating to the target plasmas using power law scalings based on the data sets obtained during scenario development and then perform NBH deposition and fast ion slowing down calculations using the ASCOT Monte Carlo orbit code [10,11] on the target plasmas to infer the DT neutron rates and fusion power. Synergetic effects between NBH and ICRH cannot be included in this approach, but previous TRANSP calculations in the conditions of JET scenarios have shown them to be small, typically affecting the neutron rates by less than $10 \%[12]$.

ASCOT has been used at JET since the late 1990's and was extended with a fusion product module AFSI 
$[13,14]$, which computes nuclear reactivities based on the fast ion distributions produced by ASCOT, as well as a range of synthetic diagnostics. ASCOT has been systematically benchmarked against the NUBEAMTRANSP [15] code, producing very similar results [16]. For the work presented here, the input for ASCOT is taken from the JETPEAK [17,18] database and analysis environment. The JETPEAK database currently includes over 15000 samples, of which more than 13000 from JET with an ITER-like wall (JET-ILW), from stationary phases and more than 1000 structured variables, from diagnostics data and from a variety of modelling codes. The duration of the stationary phases is typically in the range 0.4-3 s, depending on conditions. All values quoted throughout the paper are values taken during these stationary intervals, irrespective of what means were applied to achieve them (e.g. a plasma current "overshoot" in the ramp-up phase of the hybrid scenario). As the semi-empirical modelling presented here does not resort to a physics basis for transport and confinement, it is much faster and capable of handling much larger datasets than is possible with integrated modelling approaches. This allows performing an ASCOT calculation in batch mode for one sample (defined by a time window of stationary conditions) in approximately 10 minutes on the computers of the JET analysis cluster. Approximately 350 ASCOT calculations were performed for this work over the course of a week. This approach does not provide estimates of the likely duration of high performance phases, which are often limited by the influx of impurities and/or the onset of deleterious magnetohydrodynamic activity. The highest DD neutron rate averaged over a period of 5 seconds was $3.3 \times 10^{16} \mathrm{n} / \mathrm{s}$ for a hybrid scenario and $3.12 \times 10^{16} \mathrm{n} / \mathrm{s}$ for a baseline plasma, i.e about $60 \%$ of neutron rates when averaged over shorter $(\leq 1 \mathrm{~s})$ periods of near-stationary peak performance, as reported on here.

\section{SCALING OF DEUTERIUM PLASMA PARAMETERS}

From the JETPEAK database, subsets in baseline (q95 3, $\left.\mathrm{I}_{\mathrm{p}} \leq 4 \mathrm{MA}\right)$ and hybrid $\left(\mathrm{q}_{95} \approx 4-4.5, \mathrm{I}_{\mathrm{p}} \leq 3.1 \mathrm{MA}\right)$ discharges performed from 2016 to 2020 have been selected for extrapolation. There are significant differences between the two scenarios [1,2]. The baseline scenario consists of relaxed, sawtoothing, ELMy discharges (often mixed type, irregular and high frequency) at high $\mathrm{I}_{\mathrm{p}}$ and moderate normalised plasma pressure $\beta_{\mathrm{N}}$ in order to take advantage of the favourable energy confinement scaling with $\mathrm{I}_{\mathrm{p}}$. In the hybrid scenarios, which have type I ELMs, the plasma current is lower $(<3.1 \mathrm{MA})$, the current profile is optimised for confinement using a specially designed plasma current ramp-up procedure with a current "overshoot" resulting in a fairly high normalised plasma pressure $\beta_{\mathrm{N}}$ during the subsequent current flat-top phase. We therefore expect these differences to lead to differences in the scaling of energy and particle confinement and hence have established separate scalings, shown in figs. 1 and 2.

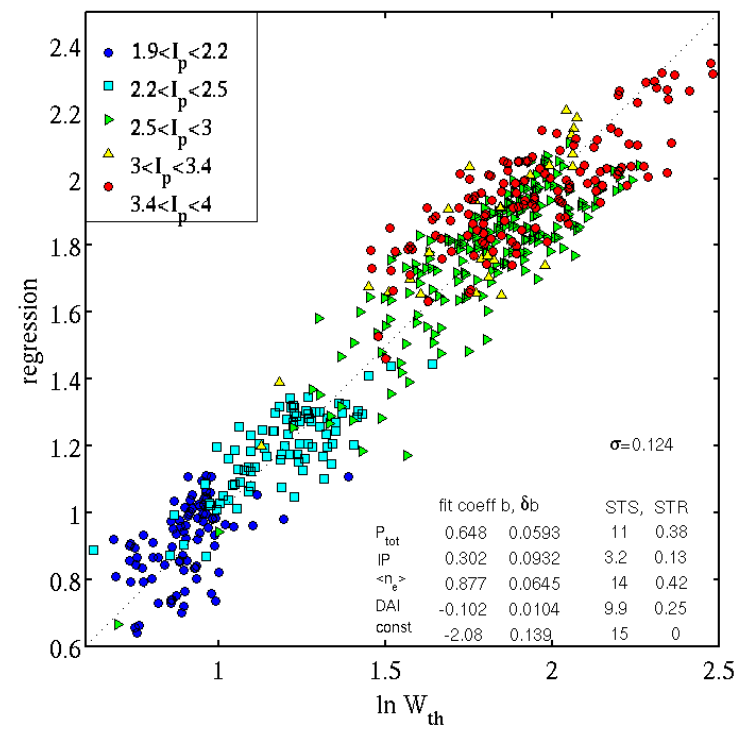

Fig. 1 Regression of thermal energy (MJ) in baseline plasmas produced during baseline development, with samples distinguished by classes of plasma current in MA. (The legend is explained in the main text.)

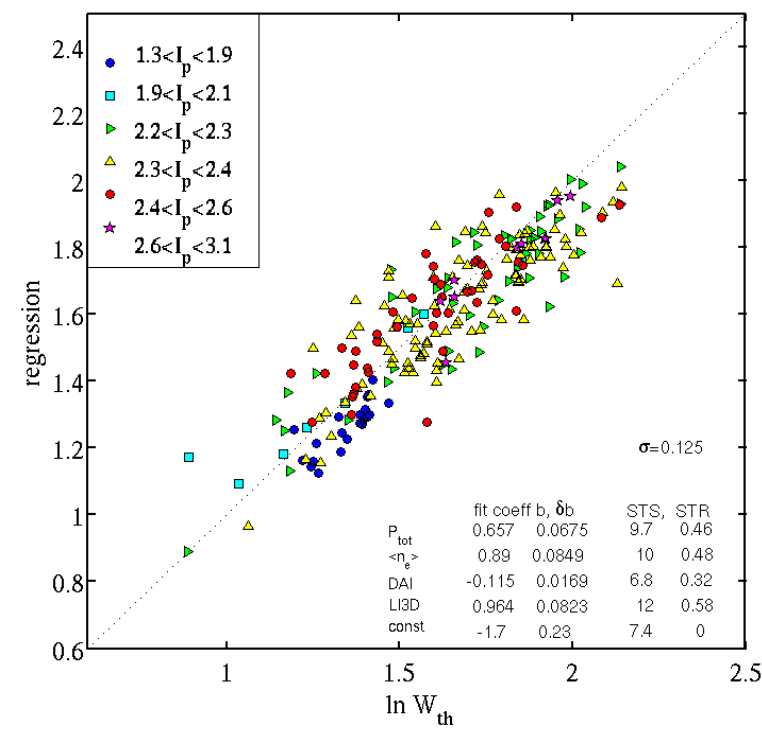

Fig.2 Regression of thermal energy (MJ) in hybrid scenario plasmas produced during hybrid development, with samples distinguished by classes of plasma current in MA. The legend is explained in the main text.

Ion temperature $\left(\mathrm{T}_{\mathrm{i}}\right)$ profiles from charge exchange spectroscopy were often only available for some of the better performing discharges. Fortunately, we were able to make use of a tight relationship between the ion temperature $\mathrm{T}_{\mathrm{i}}$ and rotation measurements on a line-integrated $\mathrm{Ni}^{26+}$ line by $\mathrm{X}$-ray crystal spectroscopy and the 
local $T_{i}$ measured by CXS in a fraction of the samples at the position of maximum emission of the Ni line. This allowed us to provide surrogate $T_{i}$ profiles as detailed in refs $[17,18]$ and calculate the plasma thermal energy for all cases, even when CXS was not available. The electron temperature $T_{e}$ and density $n_{e}$ were obtained from Thomson scattering and the ion density $n_{i}$ was inferred from $n_{e}$ and the effective charge $Z_{\text {eff }}$ inferred from visible Bremsstrahlung. The impurity profile was assumed to be entirely due to Nickel and proportional to the electron density profile, with the Nickel concentration adjusted to match $Z_{\text {eff. }}$ The regressions shown in figs 1 and 2 and expressed in eq.(1) and eq.(2) are the best 4 term regressions in terms of standard deviation obtained by automatic regressions using a multitude of regression test variables. The columns in the legend provide the exponents (b), their errors $(\delta b)$, the statistical significance $(b / \delta b)$ and the normalised statistical relevance, noted STR, which is a measure of the contribution of a regression variable to the variations of the target variable [19]. The regression variables in the legends are the total power noted $\mathrm{P}_{\text {tot }}$, the volume average density $\left\langle\mathrm{n}_{\mathrm{e}}\right\rangle$, the plasma current denoted IP in fig.2, a measure of the divertor Balmer alpha emission (DAI), noted $\Gamma$ in the equations below, and the internal inductance $1_{i}$ calculated by EFIT (denoted LI3D), which is a measure of the peakedness of the current profile. The importance of fuelling was brought to light in a study of the effects of plasma isotope composition on energy, momentum and particle confinement [9]. In that study, the line integrated divertor Balmer alpha emission was found to be a good proxy for the effective fuelling rate, including the generally dominant fuelling from recycling (see section 5.3 in [9]).

Baseline scenario scaling:

$$
W_{t h} \propto P_{\text {tot }}^{0.65}<n_{e}>^{0.88} I_{p}^{0.27} \Gamma^{-0.11}
$$

Hybrid scenario scaling :

$$
W_{t h} \propto P_{\text {tot }}^{0.69}<n_{e}>^{0.88} \Gamma^{-0.13} l_{i}^{0.92} \text { eq. } 2
$$

For the baseline scenarios, which were generally stationary for 3-5 seconds, often more than one sample was taken per pulse. The performance of many of the early hybrid scenarios degraded after an initial high confinement phase lasting $\sim 1$ second. The data set in fig. 2 and used for eq.2, therefore only include the initial high performance phase. The scaling in eq.1 differs from the multi-machine ELMy H-mode scaling [6] by a weaker $\mathrm{I}_{\mathrm{p}}$ scaling and a stronger $<\mathrm{n}_{\mathrm{e}}>$ scaling. Such differences are not surprising and are not mutually contradictory, as the dataspace visited during scenario development is highly correlated; in particular $<\mathrm{n}_{\mathrm{e}}>$ and $\mathrm{I}_{\mathrm{p}}$ are nearly proportional to each other. For ASCOT calculations the profiles of $T_{i}, T_{e}, n_{e}$, the hydrogenic ion density profiles $n_{D}$ and $n_{T}$, as well as an optional impurity density profile $\mathrm{n}_{\mathrm{z}}$ are required. These too need to be scaled. The average electron density is found to scale as

$$
<n_{e}>\propto I_{p}^{0.94} \Gamma^{-0.06} \text { eq.3a and eq.3b }<n_{e}>\propto I_{p}^{0.76} \Gamma^{-0.04}
$$

respectively for the baseline and hybrid sets. For the purpose of extrapolations to conditions with increased stored energy and density, we also need to scale the $T_{i} / T_{e}$ ratio. This is found to be weakly dependent of power, density and current for both scenarios:

$$
T_{i} / T_{e} \propto\left(P_{t o t} /<n_{e}>\right)^{0.25} I_{p}^{-0.28} \text { eq.4 }
$$

In equations 1-3, $\Gamma$ and $l_{i}$ are dependent on operational conditions and scenario execution. For the purpose of our extrapolations we simply assume that they will be reproduced identically in DT and hence we do not attempt to scale them to DT conditions.

\section{ASCOT CALCULATIONS FOR BEST PERFORMING DEUTERIUM PULSES}

We have selected the JETPEAK samples from the 10 baseline pulses and 15 hybrid pulses with the highest DD neutron rates obtained during scenario development. The baselines selected had plasma currents in the range 3-4 MA and total heating powers in the range 31-36 MW. The currents and powers for the hybrid scenarios were in the ranges 2.1-2.5 MA and 29-36 MW. These measured neutron rates in these sets are compared against ASCOT modelling in fig.3. The DD neutron rate predictions for hybrids scenarios are in good agreement with measured neutron rates, whether surrogate $T_{i}$ profiles or profiles obtained from CXS are used. For half of the baselines the DD neutron rates are strongly overpredicted (blue squares). This is not unusual, even when $T_{i}$ profiles from CXS are available [19]. No satisfactory explanation for this deficit was found in ref [20]. A likely explanation in the present baseline dataset may be that the surrogate $T_{i}$ profiles may be over-estimates of the actual ion temperatures. We have therefore repeated the ASCOT calculations with the assumption $T_{i}=T_{e}$ (blue triangles). While for all high performance discharges for which CXS based ion temperatures are available $T_{i}>T_{e}$ 
throughout the cross section, this provides a clear lower bound. For these, the ASCOT calculations underpredict the neutron rates, except at the lowest neutron rates. We believe that some of the crystal spectrometer based ion temperature profiles in the baseline set are overestimates, while the assumption $T_{i}=T_{e}$ underestimates the real ion temperatures. We show in section 5 how the impact of this discrepancy on the extrapolated DT neutron rates can be mitigated.

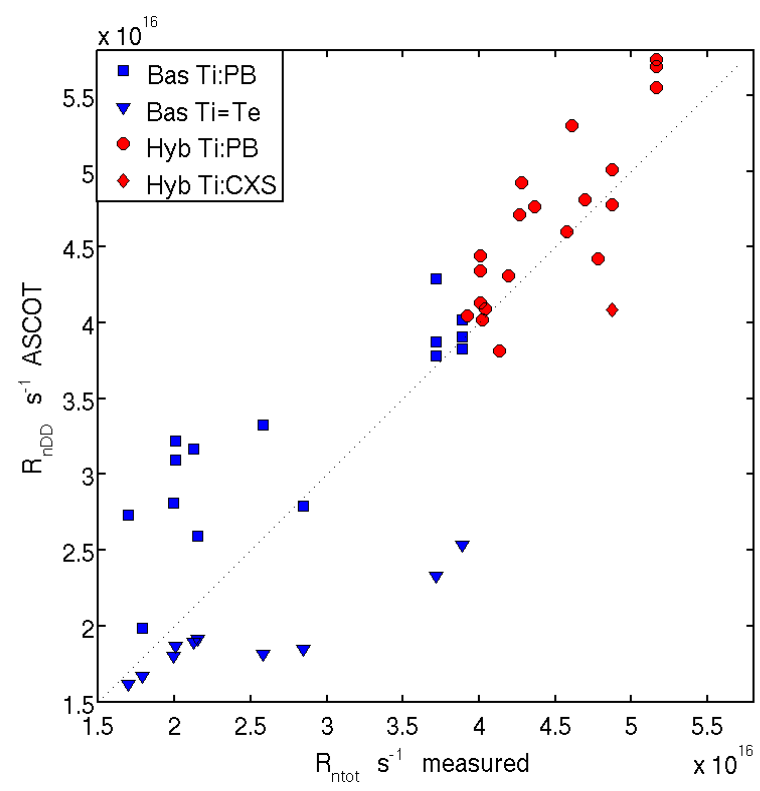

Fig.3. ASCOT calculated neutron rates vs measured neutron rates in deuterium plasmas for best performing baselines (blue) and hybrids (red). The squares and dots correspond to usage of 'surrogate' $T_{i}$ profiles derived from $X$-ray crystal spectrometry, the triangles to the assumption $T_{i}=T_{e}$. The hexagram corresponds to $T_{i}$ obtained from $C X S$.

\section{EXTRAPOLATION TO DEUTERIUM-TRITIUM CONDITIONS}

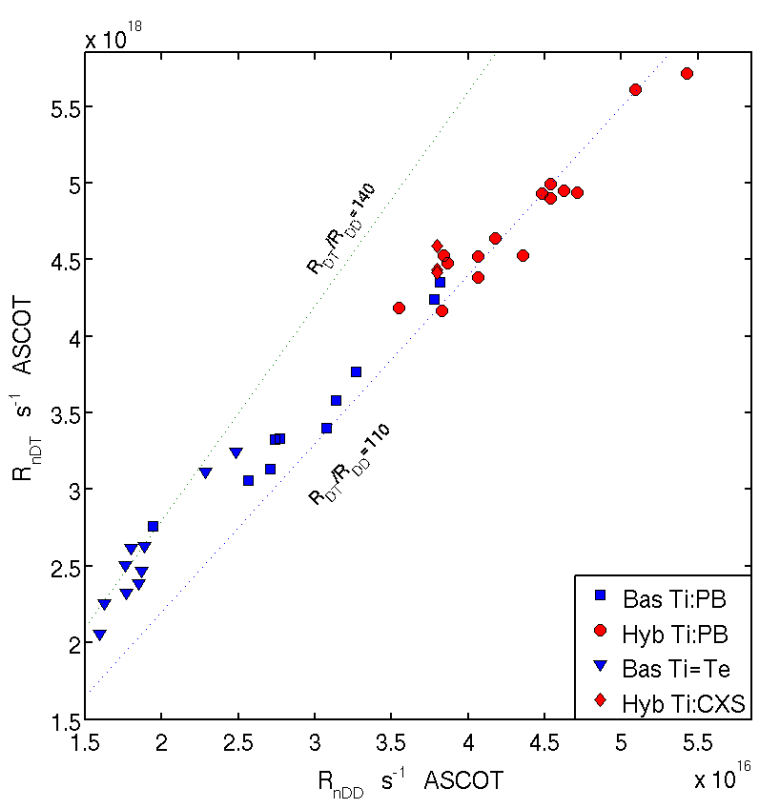

The most basic extrapolation consists in assuming that $\mathrm{T}_{\mathrm{i}}, \mathrm{T}_{\mathrm{e}}, \mathrm{n}_{\mathrm{e}}, \mathrm{n}_{\mathrm{z}}$ and $\mathrm{P}_{\text {tot }}$ are unchanged in DT plasmas, but $n_{D}=n_{T}$ and one of the two $\mathrm{NBH}$ injectors is converted to operate in tritium. Both injectors were assumed to operate at their maximal injection energy for DT, even though in the deuterium plasmas they were lower (typically 95-120keV). Fig.4 compares the calculated DD neutron rate to the calculated DT neutron rates. The ratios of DT to DD neutron rates in the range $110-140$, which is a little $(\sim 10-40 \%)$ higher than simply expected from the ratios of the DT to DD cross sections for neutron production in the relevant energy range.

Fig.4 Comparison of DD and DT neutron rates for same plasma and NBH parameters, as calculated by ASCOT, except for hydrogenic isotope composition.

Fig.5a shows the calculated DT neutron rates assuming identical temperature and density profiles, same plasma current and power versus the experimental DD neutron rates. The NBI injection energies were assumed to be at their nominal values (125keV for $\mathrm{D}$ beams, $118 \mathrm{keV}$ for $\mathrm{T}$ beams). The best performing hybrid scenario pulses have predicted fusion powers close to the objective of $15 \mathrm{MW}$, which corresponds to a DT neutron rate of $5.3 \times 10^{18} \mathrm{n} / \mathrm{s}$, shown as a red dotted horizontal line. The baselines remain clearly below this objective, especially if we assume $\mathrm{T}_{\mathrm{i}}=\mathrm{T}_{\mathrm{e}}$ (triangles) instead of the surrogate ion temperatures (squares). JET experiments in deuterium and hydrogen have shown a rather strong scaling of energy confinement with effective mass $A_{\text {eff }}$ $\left(\mathrm{A}_{\mathrm{eff}}=2.5\right.$ in the DT mixtures assumed here $), \mathrm{W}_{\mathrm{th}} \propto \mathrm{A}_{\mathrm{eff}}^{0.4-0.5}[8,9]$. We conservatively adopt the scaling as $\mathrm{W}_{\text {th }} \propto \mathrm{A}_{\text {eff }}^{0.4}$. In fig.5b we show with magenta (hybrids) and cyan (baselines) symbols the case when only the temperatures are scaled. This leads to a clear increase in neutron rates, with $\mathrm{R}_{\mathrm{nDT}}$ close to $5 \times 10^{18} \mathrm{n} / \mathrm{s}$ for the best baselines. In ref [9] it was also found that the scaling exponents for energy and particle confinement are the same. This leads to an increase in density. We show the corresponding calculations, which are hence at constant temperatures, with red and blue symbols. For hybrids, a slight reduction of DT neutron rates is the result. This reduction is more substantial for the baselines. 
Fig.5c shows the extrapolation to the target conditions $\left(\mathrm{P}_{\text {tot }}=40 \mathrm{MW}, \mathrm{I}_{\mathrm{p}}\right.$ as in $\mathrm{D}$ plasmas for hybrids, 4MA for baselines). For every discharge in the datasets described above, an extrapolated DT counterpart is constructed as by scaling the plasma energy, density and temperature ratio as in eq.1-5 and assuming that the stored energy scales as $\mathrm{A}_{\text {eff }}^{0.4}$.
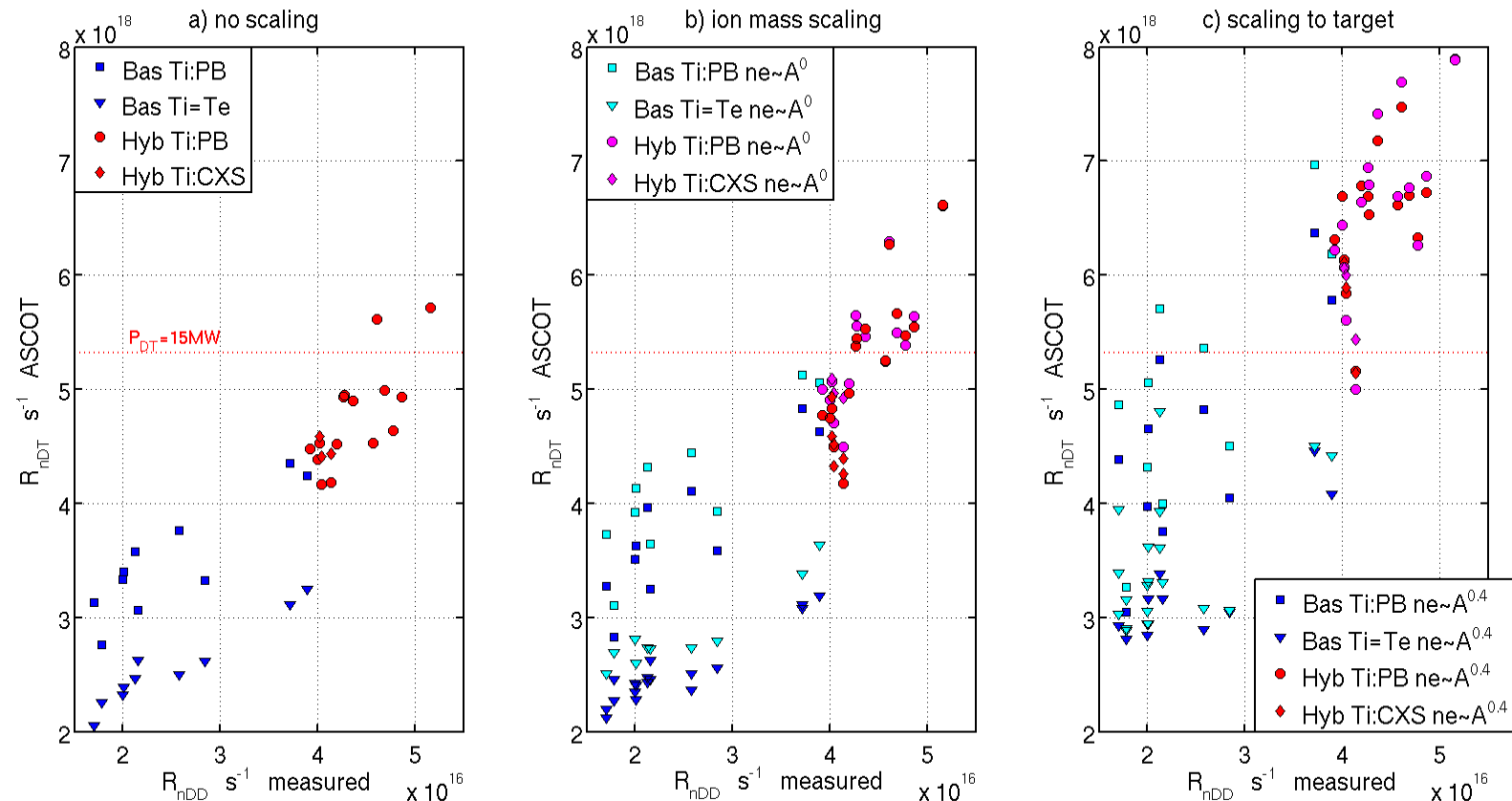

Fig. 5 Extrapolated DT neutron rates vs measured DD neutron rates in deuterium plasmas.

a) Assuming $P_{\text {tot }}, I_{p}, T_{i}, T_{e}, n_{e}$ and $n_{z}$ profiles are as in the deuterium plasmas.

b) Assuming $P_{\text {tot }}, I_{p}$ as in deuterium plasmas and $W_{t h} \propto A_{\text {eff }}^{0.4}$. The legends of subplots b) and c) apply to both. The magenta and cyan symbols are for the assumption of constant density, the blue and red ones correspond to the assumption $W_{\text {th }} \propto A_{\text {eff }}^{0.4}$ and $n_{e} \propto A_{\text {eff }}^{0.4}$, i.e. a rise in density at constant temperatures. ( $A_{\text {eff }}$ is noted $A$ for compactness)

c) Profiles scaled to target conditions ( $P_{\text {tot }}=40 \mathrm{MW}, I_{p}$ as in D plasmas for hybrids, 4MA for baselines). The red, magenta, blue and cyan symbols have the same meaning as in figs a) and b).

All subplots: The squares and circles indicate that for $T_{i}$ a surrogate based on $X$-ray crystal spectroscopy was used, triangles indicate that $T_{i}=T_{e}$ was assumed. The diamonds indicate that $T_{i}$ was obtained from CXS. In all three subplots, the DT neutron rate corresponding to a DT fusion power of 15MW is also shown as a dotted red line.

The scalings for the extrapolated temperatures, $T_{\text {iext }}=f_{i} T_{i 0}$ and $T_{\text {eext }}=f_{e} T_{e 0}$ where subscript ' 0 ' designates the original profiles and 'ext' the extrapolated ones, the required scaling multipliers $f_{i, e}$ are obtained as

$\mathrm{f}_{\mathrm{e}}=\mathrm{a}_{\mathrm{w}} \mathrm{a}_{\mathrm{n}}^{-1}\left(\mathrm{~T}_{\mathrm{e} 0}+\mathrm{T}_{\mathrm{i} 0}\right) /\left(\mathrm{T}_{\mathrm{e} 0}+\mathrm{a}_{\mathrm{T}} \mathrm{T}_{\mathrm{i} 0}\right) \quad$ eq.5 5 and $\mathrm{f}_{\mathrm{i}}=\mathrm{a}_{\mathrm{T}} \mathrm{f}_{\mathrm{e}} \quad$ eq.6

where $\mathrm{a}_{\mathrm{w}}=\mathrm{W}_{\text {thext }} / \mathrm{W}_{\text {th0 } 0}, \mathrm{a}_{\mathrm{n}}=\mathrm{n}_{\text {eext }} / \mathrm{n}_{\mathrm{e} 0}$ and $\mathrm{a}_{\mathrm{T}}=\mathrm{T}_{\text {iext }} \mathrm{T}_{\mathrm{i} 0} /\left(\mathrm{T}_{\text {eext }} \mathrm{T}_{\mathrm{e} 0}\right)$

The magenta and cyan symbols make the assumption of no additional scaling of the density with $\mathrm{A}_{\text {eff }}$ i.e. the density is scaled only according to eq. $3 \mathrm{a}$ and eq.3b. These calculations lead to the highest DT neutron rates. The results from the additional assumption $\mathrm{n}_{\mathrm{e}} \propto \mathrm{A}_{\text {eff }}^{0.4}$, in addition to the scaling in eq.4, are shown in red and blue. They are generally slightly lower for hybrids and more significantly lower for baselines. The results can be understood from the differences in ion temperatures between the hybrid and baselines scenarios, shown in fig.6.

The core ion temperatures in baseline remain, with two exceptions, below $10 \mathrm{keV}$, while they are clearly above in hybrid scenarios, reaching $15 \mathrm{keV}$ in the $\mathrm{D}$ reference plasmas and $18 \mathrm{keV}$ for the extrapolations to $40 \mathrm{MW}$. The densities are in an inverse relationship, $6 \times 10^{19}<\mathrm{n}_{\mathrm{e}}(0)<9 \times 10^{19} \mathrm{~m}^{-3}$ for the hybrids and $9 \times 10^{19}<\mathrm{n}_{\mathrm{e}}(0)<14 \times 10^{19} \mathrm{~m}^{-3}$ for the baseline scenarios. The highest neutron rates correspond largely to the lowest densities. The ratio of thermal DT to total DT neutrons is near 0.45 for the best performing baselines and hybrids, when no scaling is assumed (corresponding to fig.5a), near 0.5 when only ion mass scaling is assumed (corresponding to fig.5b), rising to 0.6 for the best baselines when extrapolated to $\mathrm{I}_{\mathrm{p}}=4 \mathrm{MA}, \mathrm{P}_{\text {tot }}=40 \mathrm{MW}$ and to near 0.55 for the best hybrids at $\mathrm{P}_{\text {tot }}=40 \mathrm{MW}$ (corresponding to fig.5c). In the hybrid scenario, a substantial portion of the core plasma is in the ideal domain, 10 $\mathrm{keV}<\mathrm{T}_{\mathrm{i}}<20 \mathrm{keV}$, where the thermal fusion reactivity $\langle\sigma \mathrm{V}\rangle_{\mathrm{DT}}$ scales as $\mathrm{T}_{\mathrm{i}}^{2}$, i.e. the thermal fusion power density $\mathrm{p}_{\mathrm{DT}}=\mathrm{n}_{\mathrm{D}} \mathrm{n}_{\mathrm{T}}<\sigma \mathrm{V}>\mathrm{DT}$ depends only on plasma ion pressure $\mathrm{p}_{\mathrm{i}}$, scaling as $\mathrm{p}_{\mathrm{i}}^{2}$. In this temperature range the thermal fusion power maximises for a given ion pressure and a trade-off between density and temperature at constant pressure is inconsequential. 
For low ion temperatures like those of the baseline scenarios in JET, the benefit of an increase in density is more than offset by the reduction in temperatures. This observation has also been made with DT predictions using integrated modelling [21-24]. For the baseline set we have therefore also performed calculations assuming that the plasma current is kept equal to the one in the deuterium discharges and only the power is raised to $40 \mathrm{MW}$. In these cases, shown in fig. 7 by white $\left(\mathrm{n}_{\mathrm{e}} \propto \mathrm{A}_{\text {eff }}{ }^{0}\right)$ and yellow $\left(\mathrm{n}_{\mathrm{e}} \propto \mathrm{A}_{\text {eff }}^{0.4}\right)$ symbols, the DT neutron rates are 10-15\% higher than at 4MA, thanks to a lower plasma density. We therefore do not recommend attempting to raise the plasma currents in baseline plasmas to 4MA. Repeating them at the currents achieved in the deuterium discharges, i.e. $\mathrm{I}_{\mathrm{p}}=3.5 \mathrm{MA}$ for the two baseline cases with the highest predicted neutron rates, provides higher fusion power at lower risk.
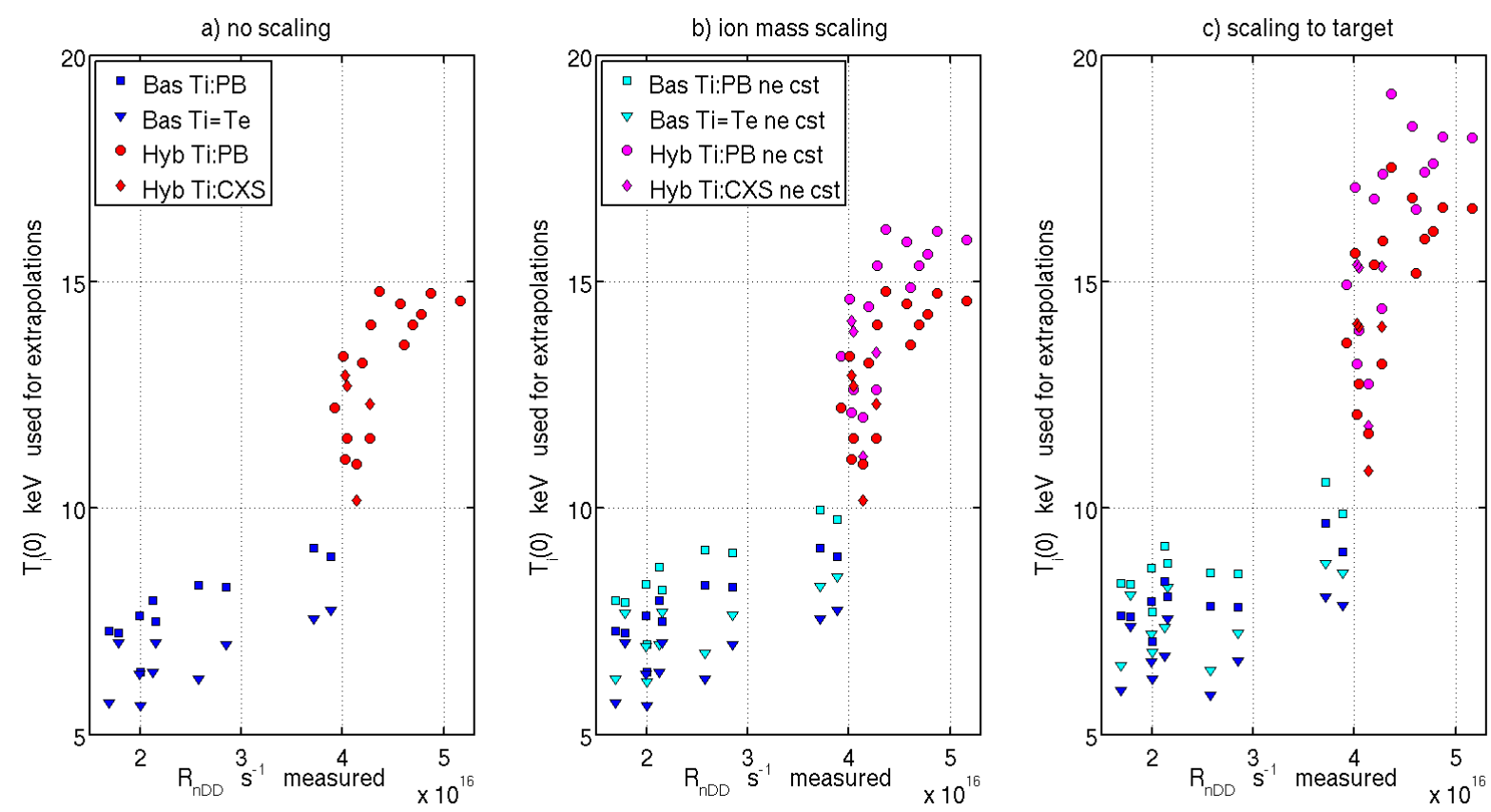

Fig.6 Central ion temperatures used for the ASCOT calculations. The symbols have the same meaning as in fig.5.

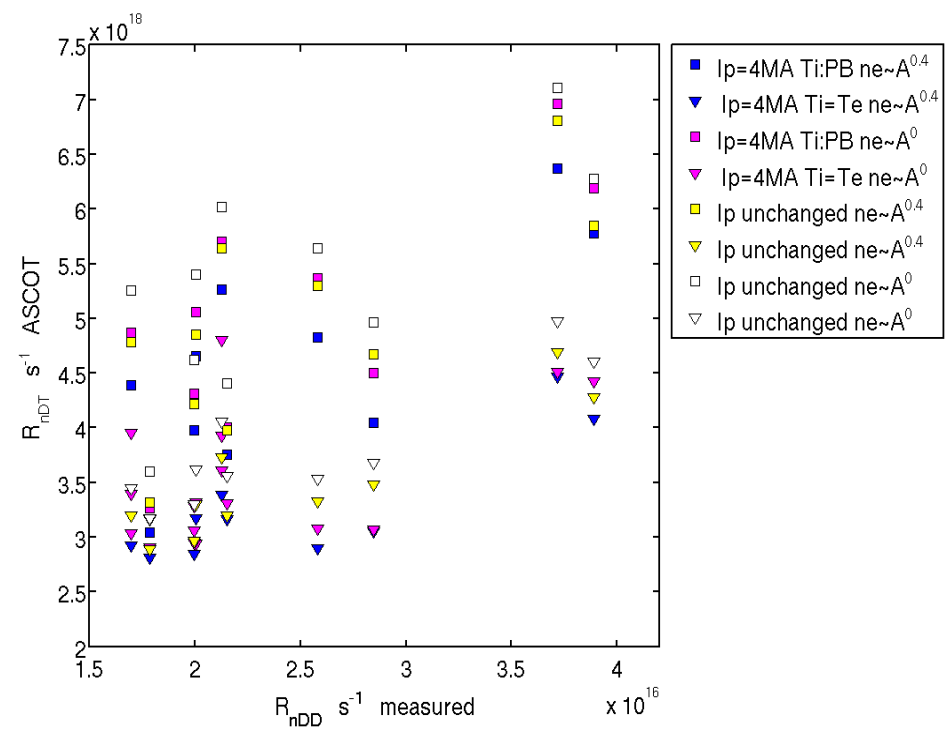

Fig. 7 Comparison of baseline DT neutron rates for $P_{\text {tot }}=40 \mathrm{MW}$ assuming $I_{p}=4 \mathrm{MA}$ (blue and magenta, as in fig.5c) and assuming $I_{p}$ is kept the same as in the corresponding deuterium plasmas. Yellow symbols correspond to the assumption $W_{\text {th }} \propto A_{\text {eff }}^{0.4}$ and $n_{e} \propto A_{\text {eff }}^{0.4}$, white ones to $W_{\text {th }} \propto A_{\text {eff }}^{0.4}$ and $n_{e} \propto A_{\text {eff }}^{0}$. The squares and triangles have the same meaning as in the previous figures. 


\section{RENORMALISATION AND ALPHA PARTICLE HEATING}

While the nominally installed total plasma heating power at JET is $40 \mathrm{MW}$, the highest power achieved on a sustained basis was 38MW. For most of the scenario development phase, the power delivered to the plasma was below 35MW. This is easily understood as being a result of the finite technical availability of each of the 20 heating subsystems. As an example, assuming each subsystem independently has an availability of $80 \%$, the probability of all being available simultaneously is $1.15 \%$, while the probability of at least 16 of them being available is $63 \%$. We hence consider the extrapolations to full target conditions (fig.5c) as unrealistic. We believe that the assumption of a power availability being the same in DTE2 as it was during the preparation period, i.e. as in fig.5b, to be realistic, i.e. $\sim 30 \mathrm{MW}$ from $\mathrm{NBH}$ and up to 5MW for ICRH. We also consider that assuming that the density will scale as $\mathrm{n}_{\mathrm{e}} \propto \mathrm{A}_{\text {eff }}^{0.4}$ to be more realistic (and more conservative) than the assumption that the density can be kept at the same value as in the deuterium discharges. We now examine the uncertainties arising from $T_{i 0}$ and the effect of alpha particle heating. A way to reduce uncertaintainties on the calculated neutron rates is to use the ratio of calculated to measured DD neutron rates as a renormalisation factor, i.e.

$R_{n D T} *=R_{n D T \text { calc }} \frac{R_{n D D \text { meas }}}{R_{n D \text { D calc }}}$ eq.7

The differences between the renormalised DT neutron rates, shown in fig.8a, are far less dependent on the assumed ion temperatures than shown in fig. $5 \mathrm{~b}$. These calculations also do not include the heating provided by fusion alpha particles, the power of which ranges from 1.1 to 3.7 MW. First orbit loss calculations show that some $85 \%$ of the alphas are confined in hybrid scenarios for $\mathrm{I}_{\mathrm{p}}=2.3 \mathrm{MA}$ and $90 \%$ for baselines at $\mathrm{I}_{\mathrm{p}} \geq 3 \mathrm{MA}$. Alphas, like minority ICRH schemes, heat the electrons mostly, but nonetheless contribute to raising the $T_{i}$ indirectly by raising $T_{e}$. This reduces the equipartition heat exchange between ions and electrons, allowing the $T_{i}$ to rise also, thereby increasing the thermal DT neutron rate. Another possible effect, the reduction of the ion heat diffusivity by stabilisation of Ion Temperature Gradient turbulence is outside of the scope of our empirical approach [24,25]. Fig. 8b, shows the neutron rates that can realistically be expected in DTE2 on the basis of our modelling assumptions when the heating by confined alpha particles is included.

The DTE2 expectations expressed in fig.8b for realistic conditions of power availability are clearly encouraging, as all of the hybrid scenario plasmas examined achieve DT neutron rates close to, or above, the target of $5.3 \times 10^{18} \mathrm{~s}^{-1}$, corresponding to $15 \mathrm{MW}$ of fusion power. The highest of the hybrid prediction corresponds to a DT fusion power of $19 \mathrm{MW}$. The two best baselines have predicted DT fusion power in the range 14-16.6 MW, depending on the assumptions.
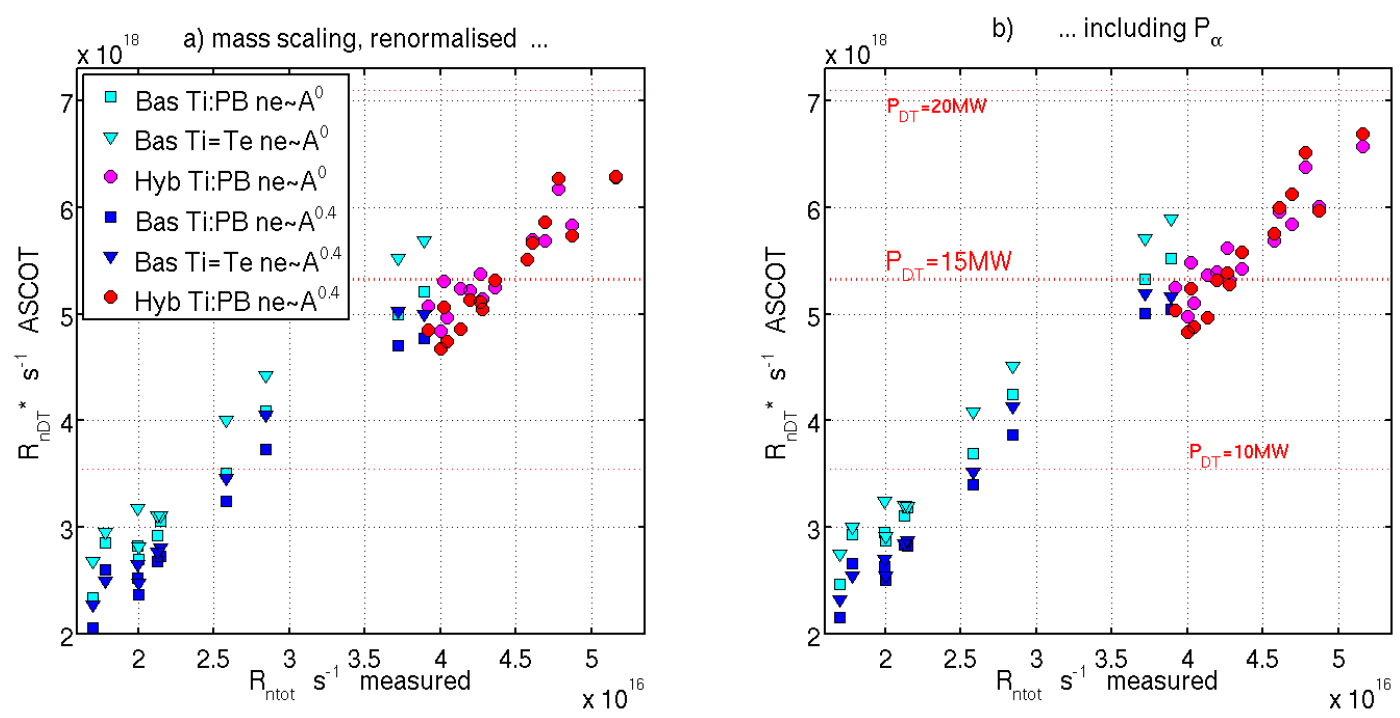

Fig.8. a) Expected neutron rates assuming the plasma currents and heating powers are kept unchanged from the deuterium plasmas, after normalisation (eq.8), b) as a), but including heating by confined alpha particles. 


\section{ACKNOWLEDGEMENTS}

We are grateful to Dr. J. Mailloux for a thorough pre-submission review of this paper. This work has been carried out within the framework of the EUROfusion Consortium and has received funding from the Euratom research and training programme 2014-2018 and 2019-2020 under grant agreement No 633053. The views and opinions expressed herein do not necessarily reflect those of the European Commission.

\section{REFERENCES}

[1] J. Mailloux et al. submitted to Nuclear Fusion Special issue: Overview and Summary Papers from the 28th Fusion Energy Conference (Nice, France, 10-15 May 2021)

[2] L. Garzotti et al. 2019 Nucl. Fusion 59076037

[3] J. Garcia et al. 2019 Nucl. Fusion 5908604

[4] J. Garcia et al. 28th IAEA FEC, 2021, IAEA-EX/1-2

(Oral, 8 page paper, IAEA address still unknown)

[5] E.J. Doyle et al 2007, ITER Physics Basis, Nucl. Fusion 47 S18

[6] G. Verdoolaege et al 2021 Nucl. Fusion 61076006

[7] P. Sirén et al, 28th IAEA FEC, https://conferences.iaea.org/event/214/contributions/17223/

(Poster, 8 page paper, IAEA address still unknown)

[8] Maggi C.F. et al 2018 Plasma Phys. Control. Fusion 60014045

[9 ] H. Weisen et al, Journal of Plasma Physics, vol 86, 905860501

[10] J. A. Heikkinen et al. 2001 J. Comput. Phys. 173 527-48

[11] E. Hirvijoki et al. 2014 Comput. Phys. Commun. 185 1310-1321

[12] K.K. Kirov et al 2021 Nucl. Fusion 61046017

[13] P. Sirén et al. 2018 Nucl. Fusion 58016023

[14] P. Sirén et al. 2019 JINST 14 C11013

[15] A. Pankin et al. 2004 Comput. Phys. Commun. 159 157-184

[16] P. Sirén et al., subm. to Nucl. Fusion 2021

[17] H. Weisen et al. 2020 Nucl. Fusion 60036004

[18] H. Weisen et al, 28th IAEA FEC, 2021, https://conferences.iaea.org/event/214/contributions/17320/

(Poster, 8 page paper, IAEA address still unknown)

[19] O.J.W.F. Kardaun, 2005 Classical Methods of Statistics (Berlin: Springer) ISBN 3540292888, 9783540292883

[20] H. Weisen et al 2017 Nucl. Fusion 57076029

[21] Garcia et al 2015 Nucl. Fusion 55053007

[22] J Garcia et al 2017 Plasma Phys. Control. Fusion 59014023

[23] F.J. Casson et al 2020 Nucl. Fusion 60066029

[24] J Garcia et al PHYSICS OF PLASMAS 25, 055902 (2018)

[25] D. Testa and M. Albergante 2012 Nucl. Fusion 520830010 